\title{
Application in the STRATHE trial of a score system to compare the efficacy and the tolerability of different therapeutic strategies in the management of hypertension
}

\author{
Bernard Waeber' \\ Jean-Jacques Mourad ${ }^{2}$ \\ 'Division de Physiopathologie \\ Clinique, Centre Hospitalier \\ Universitaire Vaudois et Université \\ de Lausanne, Lausanne, Switzerland; \\ ${ }^{2}$ Hôpital Avicienne, Bobigny, France
}

Correspondence: Bernard Waeber CHUV, Division de Physiopathologie Clinique, MP I4, CH-I0II Lausanne, Switzerland

Tel +4I 2I 3I4 0760

Fax $+14|2| 3 \mid 42518$

Email bernard.waeber@chuv.ch

\begin{abstract}
A score system integrating the evolution of efficacy and tolerability over time was applied to a subpopulation of the STRATHE trial, a trial performed according to a parallel group design, with a double-blind, random allocation to either a fixed-dose combination strategy (perindopril/indapamide $2 \mathrm{mg} / 0.625 \mathrm{mg}$, with the possibility to increase the dose to $3 \mathrm{mg} / 0.935 \mathrm{mg}$, and $4 \mathrm{mg} / 1.250 \mathrm{mg}$ if needed, $\mathrm{n}=118$ ), a sequential monotherapy approach (atenolol $50 \mathrm{mg}$, followed by losartan $50 \mathrm{mg}$ and amlodipine $5 \mathrm{mg}$ if needed, $\mathrm{n}=108$ ), or a stepped-care strategy (valsartan $40 \mathrm{mg}$, followed by valsartan $80 \mathrm{mg}$ and valsartan $80 \mathrm{mg}+$ hydrochlorothiazide $12.5 \mathrm{mg}$ if needed, $\mathrm{n}=103$ ). The aim was to lower blood pressure below 140/90 mmHg within a 9-month period. The treatment could be adjusted after 3 and 6 months. Only patients in whom the study protocol was strictly applied were included in this analysis. At completion of the trial the total score averaged 13.1 \pm 70.5 (mean \pm SD) using the fixed-dose combination strategy, compared with $-7.2 \pm 81.0$ using the sequential monotherapy approach and $-17.5 \pm 76.4$ using the stepped-care strategy. In conclusion, the use of a score system allows the comparison of antihypertensive therapeutic strategies, taking into account at the same time efficacy and tolerability. In the STRATHE trial the best results were observed with the fixed-dose combination containing low doses of an angiotensin enzyme converting inhibitor (perindopril) and a diuretic (indapamide).
\end{abstract}

Keywords: antihypertensive therapy, tolerability, antihypertensive efficacy, fixed-dose combination, sequential monotherapy, stepped-care treatment

\section{Introduction}

During the last few decades numerous clinical trials have been performed in the field of hypertension. These trials were required for the development of new antihypertensive agents and to demonstrate their efficacy and tolerability in comparison with placebo or established blood-pressure-lowering agents. Pharmacological treatment of hypertension has been shown over the years to decrease significantly morbidity and mortality due to cardiovascular and renal diseases (Staessen et al 2003; Turnbull et al 2005; Williams 2005). Today it appears critical to achieve strict blood pressure control in hypertensive patients in order to provide maximum benefit from blood pressure lowering, and current guidelines recommend bringing blood pressure below $140 / 90 \mathrm{mmHg}$ in every patient, and even below $130 / 80 \mathrm{mmHg}$ if high blood pressure co-exists with diabetes mellitus and/or renal disease (Chobanian et al 2003; Mancia et al 2007). Different therapeutic strategies are available to reach these goal blood pressures, but increasing evidence indicates that the combination of two or more drugs is needed in most patients (Brunner et al 1990; Dusing 2006). In terms of 
pharmacological treatment of hypertension, the ideal drug regimen should be effective, well tolerated, and easy to take every day, thereby facilitating long-term adherence. To what extent a given treatment fulfills these criteria remains, however, difficult to assess in clinical trials.

The present study was aimed to evaluate whether a score system integrating the evolution of efficacy and tolerability over time would enable a better characterization of advantages and disadvantages of antihypertensive drug regimens. To this end we created a score system and applied it to the STRATHE trial, a parallel group design trial which compared in double-blind fashion different therapeutic strategies (Mourad et al 2004).

\section{Patients and methods}

The STRATHE study was performed in France by 193 community physicians in 533 patients with a mean sitting systolic blood pressure $\geq 160 \mathrm{mmHg}$ and/or a mean diastolic blood pressure $\geq 95 \mathrm{mmHg}$ after a 4 -week single-blind placebo run-in. After randomization to a fixed low-dose combination (perindopril/indapamide, $2 \mathrm{mg} / 0.625 \mathrm{mg}$, increased if required first to $3 \mathrm{mg} / 0.937 \mathrm{mg}$ and later to $4 \mathrm{mg} / 1.25 \mathrm{mg}$ ) ( $\mathrm{n}=180$ ), sequential monotherapy (atenolol $50 \mathrm{mg}$, followed as needed first by losartan $50 \mathrm{mg}$, and then by amlodipine $5 \mathrm{mg})(\mathrm{n}=176)$, or stepped care (valsartan $40 \mathrm{mg}$, increased if necessary first to valsartan $80 \mathrm{mg}$, with the possibility to combine then valsartan $80 \mathrm{mg}$ and hydrochlorothiazide, $12.5 \mathrm{mg}(\mathrm{n}=177)$ (Mourad et al 2004). In each study arm the aim was to lower blood pressure below $140 / 90 \mathrm{mmHg}$. To this end, the treatment could be adjusted as described above after 3 and 6 months of therapy. The final visit was planned after 9 months of treatment, or at 6 months if target blood pressure was achieved. Upgrading to a superior level was recommended when systolic blood pressure was $\geq 140$ and $\leq 160 \mathrm{mmHg}$ and/or diastolic blood pressure $\geq 90 \mathrm{mmHg}$, and $\leq 95 \mathrm{mmHg}$, but was mandatory when systolic pressure was $>160 \mathrm{mmHg}$ and/or diastolic blood pressure $>95 \mathrm{mmHg}$. There was, however, still the possibility, regardless of the blood pressure achieved, to maintain the previous level if the investigators were concerned about treatment upgrading, for safety reasons. Blood pressure was measured in the sitting position using a mercury sphygmomanometer. Three readings were obtained at each visit, with at least a 1-min interval between each reading, and were then averaged. All study tablets were encapsulated to conceal their identity and had to be taken once a day in the morning.

The present analysis included patients having still their blood pressure (BP) in whom the study protocol was strictly applied, which means that the treatment was modified according to the randomized therapeutic strategies each time blood pressure was still $\geq 140 / 90 \mathrm{mmHg}$ (fixed low-dose combination, $\mathrm{n}=118$; sequential monotherapy, $\mathrm{n}=108$; stepped-care approach, $\mathrm{n}=103$ ). A score system was created to assess the advantages and the disadvantages of the treatment approaches. The number of points was attributed in order to give equal importance to efficacy and tolerability in comparing the different therapeutic strategies as follows:

Month 3:

$$
\begin{aligned}
& \mathrm{BP}<140 / 90 \mathrm{mmHg}=+25 \\
& \mathrm{BP} \geq 140 / 90 \mathrm{mmHg}=-25 \\
& \text { side-effect(s) during months } 1-3=-25
\end{aligned}
$$

Month 6: $\quad \mathrm{BP}<140 / 90 \mathrm{mmHg}=+25$

$$
\mathrm{BP} \geq 140 / 90 \mathrm{mmHg}=-25
$$$$
\text { side-effect(s) during months } 3-6=-25
$$

Month 9: $\quad \mathrm{BP}<140 / 90 \mathrm{mmHg}=+50$

$$
\mathrm{BP} \geq 140 / 90 \mathrm{mmHg}=-50
$$$$
\text { side-effect(s) during months } 6-9=-25
$$

At any time: withdrawal because of side-effect(s) $=-50$

Data are reported as means $\pm \mathrm{SD}$. The scores calculated as described above were not subjected to statistical analysis since they were not predefined endpoints (Mourad et al 2004). Also, this study includes only the subset of patients who underwent changes in therapy during the trial each time their blood pressure was still $\geq 140 \mathrm{mmHg}$ for systolic and/or $\geq 90 \mathrm{mmHg}$ for diastolic.

\section{Results}

The fraction of patients who normalized their blood pressure $(<140 / 90 \mathrm{mmHg}$ ) during the 9 month follow-up was $72.9 \%$ in the fixed low-dose combination group, compared with $59.3 \%$ and $52.4 \%$ in the sequential monotherapy and steppedcare groups, respectively. Table 1 shows the percentage of patients in the three treatment groups who normalized their blood pressure during the course of the trial (Months 3, 6 and 9).

Figure 1 shows the percentage of patients exhibiting a cumulative positive, neutral (sum of positive and negative points $=0$ ), or negative score at completion of the trial. A positive score was achieved more often using the fixed lowdose strategy (64.4\%) than using the sequential monotherapy $(51.9 \%)$ or the stepped-care $(43.7 \%)$ approach.

During the course of the trial the score increased by an average of $13.1 \pm 70.5$ points in the fixed low-dose combination group, but decreased by $7.2 \pm 81.0$ and $17.5 \pm 76.4$ points in the sequential monotherapy and stepped-care groups, respectively. 
Table I Percentage of patients having their blood pressure normalized $(<\mid 40 / 90 \mathrm{mmHg})$ at months 3, 6, and 9

\begin{tabular}{lll}
\hline & $\begin{array}{l}\text { Fixed-dose } \\
\text { combination(n= I I 8) }\end{array}$ & $\begin{array}{l}\text { Sequential } \\
\text { monotherapy }(\mathbf{n}=108)\end{array}$ \\
\hline Month 3 & $59.8 \%$ & $60.4 \%$ \\
Month 6 & $69.2 \%$ & $61.5 \%$ \\
Month 9 & $72.9 \%$ & $59.3 \%$ \\
\hline
\end{tabular}

\section{Discussion}

Controlled clinical trials are critical for characterizing the efficacy and the tolerability of new antihypertensive agents. This has been done, for instance, during the developmental phase of the fixed low-dose combination containing the angiotensin converting enzyme inhibitor perindopril and the diuretic indapamide (Laurent 2001). In addition morbidity and mortality trials should be performed whenever possible to define the most appropriated indications of each class of antihypertensive drugs (Williams 2005; Zanchetti 2005). During recent years the importance of bringing blood pressure below $140 \mathrm{mmHg}$ for systolic and $90 \mathrm{mmHg}$ for diastolic has been emphasized (Chobanian et al 2003; Mancia et al 2007). To achieve these target blood pressures the co-administration of two drugs is often required, accounting for the growing interest for fixed-dose combinations (Brunner et al 1990; Dusing 2006). The advantage of combining drugs acting by different mechanisms in a single pill is to enhance the antihypertensive efficacy, but not at the expense of reduced tolerability (Law et al 2003), and to facilitate long-term persistence on treatment (Van Wijk et al 2005). There is therefore a strong rationale for the use of fixed-dose combinations not only as second-line, but also as first-line therapy (Elliott 2002; Welsh and Ferro 2004), and this view has been supported by international experts in hypertension guidelines (Chobanian et al 2003: Mancia et al 2007).

Beyond combination therapy as first step, the traditional strategies for treating hypertension with drugs comprise the stepped-care approach (monotherapy as initial treatment, followed if needed by a dose titration or the addition of a second drug) and the sequential monotherapy approach (rotation through several monotherapies from different classes until blood pressure control is reached) (Brunner et al 1990). The design of the STRATHE trial is original as it enabled, under controlled conditions, a direct comparison of different therapeutic strategies in clinical practice (Mourad

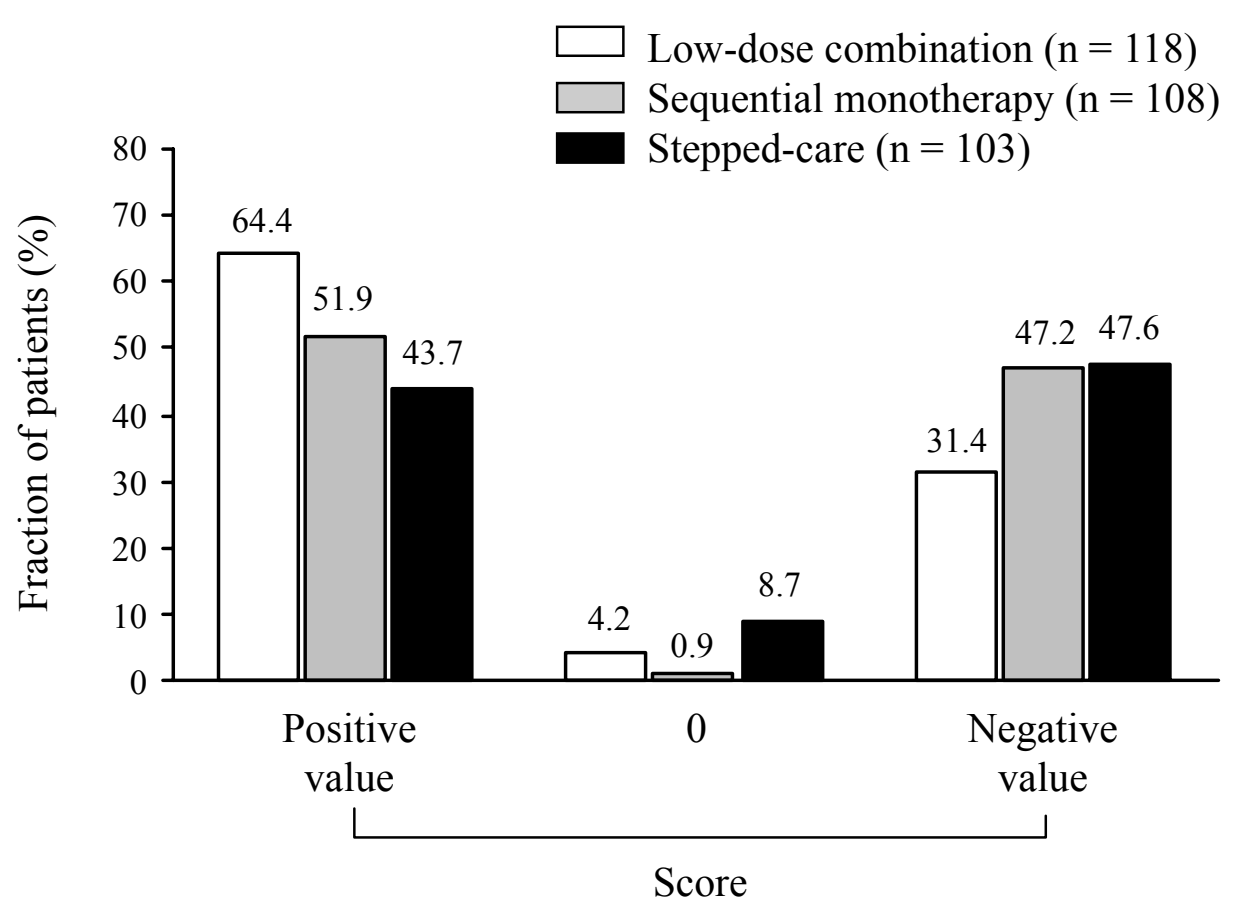

Figure I Fraction of patients exhibiting a cumulative positive, neutral or negative score at the end of the trial. 
et al 2004). A first-line management of essential hypertensive patients based on a low-dose combination of perindopril and indapamide normalized blood pressure $(<140 / 90 \mathrm{mmHg})$ in significantly more patients $(62 \%)$ than a sequential monotherapy strategy involving atenolol, losartan, and amlodipine (49\%), and a stepped-care strategy involving valsartan and hydrochlorothiazide (47\%), without difference, however, between the three groups with regard to the tolerability profile. The blood pressure normalization rate observed in the subset of patients of the STRATHE trial included in the present analysis is even better $(72.9 \%$ in the fixed low-dose combination group, $59.3 \%$ in the sequential monotherapy group, and $52.4 \%$ in the stepped-care group). These patients were selected as their treatment was modified according to the study protocol at each visit if their blood pressure was still $\geq 140 / 90 \mathrm{mmHg}$, indicating that it is important to adjust antihypertensive treatment if blood pressure remains uncontrolled.

The choice of the most appropriate strategy to treat hypertensive patients is still difficult. To be considered are efficacy or tolerability criteria, as well as the presence of target organ damage or associated cardiovascular/renal disorders. Some drug regimens may require fewer adjustments than others at initiation of treatment, an advantage derived from a better antihypertensive efficacy combined with a preserved tolerability. The turbulence resulting from treatment adjustments might have important implications. In addition to a negative economic impact, frequent drug switches might lead patients to become non-compliant, or even to stop the treatment (Hughes and McGuire 1998; Dusing 2001; Urquhart 2001). Notably, prompt blood pressure control now appears desirable in order to benefit maximally from the blood pressure lowering (Julius et al 2004). Admittedly, the current way to analyze results of clinical trials in the field of hypertension does not enable assessment of how difficult or easy it is to normalize blood pressure in the individual patient. This prompted us to test whether a score system integrating the evolution of efficacy and tolerability over time would facilitate the weighing of advantages and disadvantages of different therapeutic strategies, and therefore render comparison between them more meaningful. For this purpose we used the observations obtained in the STRATHE trial. A better score was seen in the patients allocated to the fixed low-dose combination compared with the two other options. Notably, the number of points given to efficacy and tolerability criteria could be attributed differently in other clinical trials, depending, on the relative importance one wants to give to the various criteria to be taken into account.
In summary, it is possible to analyze the results of trials aimed to compare different treatment strategies in the field of hypertension using a score system. This approach gives an integrative view on the evolution of efficacy and tolerability, and reflects how difficult or easy it is to normalize blood pressure. Further studies are, however, needed to test prospectively whether this type of analysis is more informative than an analysis based on efficacy and tolerability criteria considered separately.

\section{References}

Brunner HR, Menard J, Waeber B, et al. 1990. Treating the individual hypertensive patient: considerations on dose, sequential monotherapy and drug combinations. J Hypertens, 8:3-11.

Chobanian AV, Bakris GL, Black HR, et al. 2003. Seventh report of the Joint National Committee on Prevention, Detection, Evaluation, and Treatment of High Blood Pressure. Hypertension, 42:1206-52.

Dusing R. 2001. Adverse events, compliance, and changes in therapy. Current Hypertens Rep, 3:488-92.

Dusing R. 2006. Overcoming barriers to effective blood pressure control in patients with hypertension. Curr Med Res Opin, 22:1545-53.

Elliott WJ. 2002. Is fixed combination therapy appropriate for initial hypertension treatment? Current Hypertens Rep, 4:278-85.

Hughes D, McGuire A. 1998. The direct costs to the NHS of discontinuing and switching prescriptions for hypertension. J Hum Hypertens, 12:533-7.

Julius S, Kjeldsen SE, Weber M, et al. 2004. Outcomes in hypertensive patients at high cardiovascular risk treated with regimens based on valsartan or amlodipine: the VALUE randomised trial. Lancet, 363:2022-31.

Laurent S. 2001. Clinical benefit of very-low-dose perindopril-indapamide combination in hypertension. J Hypertens, 19(suppl 4):S9-S14.

Law MR, Wald NJ, Morris JK, et al. 2003. Value of low dose combination treatment with blood pressure lowering drugs: analysis of 354 randomised trials. BMJ, 326:1427-31.

Mancia G, De Backer G, Dominiczak A, et al. 2007. Guidelines for the Management of Arterial Hypertension: The Task Force for the Management of Arterial Hypertension of the European Society of Hypertension (ESH) and of the European Society of Cardiology (ESC). J Hypertens, 25:1105-87.

Mourad JJ, Waeber B, Zannad F, et al. 2004. Comparison of different therapeutic strategies in hypertension: a low-dose combination of perindopril/indapamide versus a sequential monotherapy or a stepped-care approach. J Hypertens, 22:2379-86.

Staessen JA, Wang JG, Thijs L. 2003. Cardiovascular prevention and blood pressure reduction: a quantitative overview updated until 1 March 2003. J Hypertens, 21:1055-76.

Turnbull F, Neal B, Algert C, et al. 2005. Effects of different blood pressurelowering regimens on major cardiovascular events in individuals with and without diabetes mellitus: results of prospectively designed overviews of randomized trials. Arch Intern Med, 165:1410-19.

Urquhart J. 2001. Some economic consequences of noncompliance. Current Hypertens Rep, 3:473-80.

Van Wijk BL, Klungel OH, Heerdink ER, et al. 2005. Rate and determinants of 10-year persistence with antihypertensive drugs. J Hypertens, 23:2101-7.

Welsh L, Ferro A. 2004. Drug treatment of essential hypertension: the case for initial combination therapy. Int J Clin Pract, 58:956-63.

Williams B. 2005. Recent hypertension trials: implications and controversies. J Am Coll Cardiol, 45:813-27.

Zanchetti A. 2005. Evidence-based medicine in hypertension: what type of evidence? J Hypertens, 23:1113-20. 